\title{
Drift velocity monitoring of the CMS muon drift chambers
}

\author{
Lars Sonnenschein* \\ RWTH Aachen University, III. Physikalisches Institut A, 52056 Aachen, Germany \\ E-mail: Lars. Sonnenschein@cern.ch \\ on behalf of the CMS collaboration
}

\begin{abstract}
The drift velocity in drift tubes of the CMS muon chambers is a key parameter for the muon track reconstruction and trigger. It needs to be monitored precisely in order to detect any deviation from its nominal value. A change in absolute pressure, a variation of the gas admixture or a contamination of the chamber gas by air affect the drift velocity. Furthermore, the temperature and magnetic field influence its value. First data, taken with a dedicated Velocity Drift Chamber (VDC) built by RWTH Aachen IIIA are presented.
\end{abstract}

XXth Hadron Collider Physics Symposium

November 16 - 20, 2009

Evian, France

* Speaker. 


\section{Introduction}

The muon drift tube chambers of the CMS detector rely crucially on the accurate knowledge of the drift velocity to reconstruct muon tracks as precise as possible. A monitor system for the direct measurement of the drift velocity is presented together with chamber gas pressure monitoring. In the first section the CMS muon drift tube chambers are explained, followed by two sections about their pressure and drift velocity monitoring, including latest measurements.

\section{CMS muon drift tube chambers}

The barrel of the CMS detector [1] consists of five wheels, each instrumented with 50 muon Drift Tube (DT) stations outside the $4 \mathrm{~T}$ solenoid magnet, arranged in four radial layers embedded in the iron return yoke of the solenoid. Each station is $29 \mathrm{~cm}$ thick and has a length along the beam axis of $2.5 \mathrm{~m}$, given by the wheel width. Depending on the layer the station width varies between two and four meters. The three inner muon stations consist of three and the outermost of two superlayers which in turn consist of four layers of drift cells. The drift cells of the innerand outermost superlayers are oriented parallel to the beam axis to measure the projection of muon tracks perpendicular to the beam axis. The middle superlayer (present in the three inner muon stations) is dedicated to the measurement of the track projection along the beam axis. Each drift cell has a cross section of $42 \mathrm{~mm} \times 13 \mathrm{~mm}$. Field shaping strips $(+1800 \mathrm{~V})$ at the inner bottom and top of the drift cell are responsible for a very homogeneous electric field between the anode $(+3600 \mathrm{~V})$ and the cathode $(-1200 \mathrm{~V})$. The drift cells are operated with a gas admixture of $\operatorname{Ar}(85 \%$ vol.) and $\mathrm{CO}_{2}(15 \%$ vol.) at slight overpressure ( $\sim 2-10 \mathrm{mbar})$. If a muon crosses a cell, ionised molecules drift to the cathode while electrons reach the anode sense wire. The crossing position of the muon can be computed by means of the drift velocity assuming linear behaviour due to a homogeneous electric field.

\section{Drift velocity monitoring}

The gas from the DT's is piped to the VDC monitoring system to check for gas anomalies. Fig. 1 shows the working principle of a VDC. It consists of a chamber equipped with anode

Figure 1: Principle and geometry of the Velocity Drift Chamber (VDC).

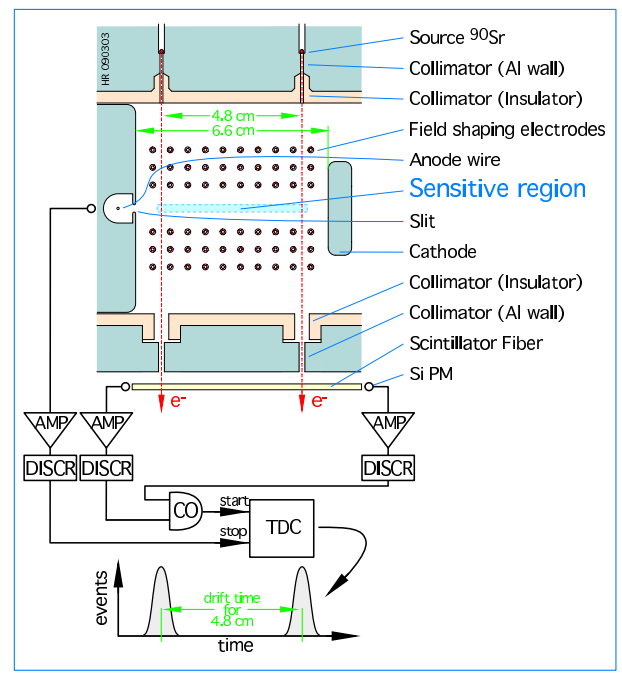



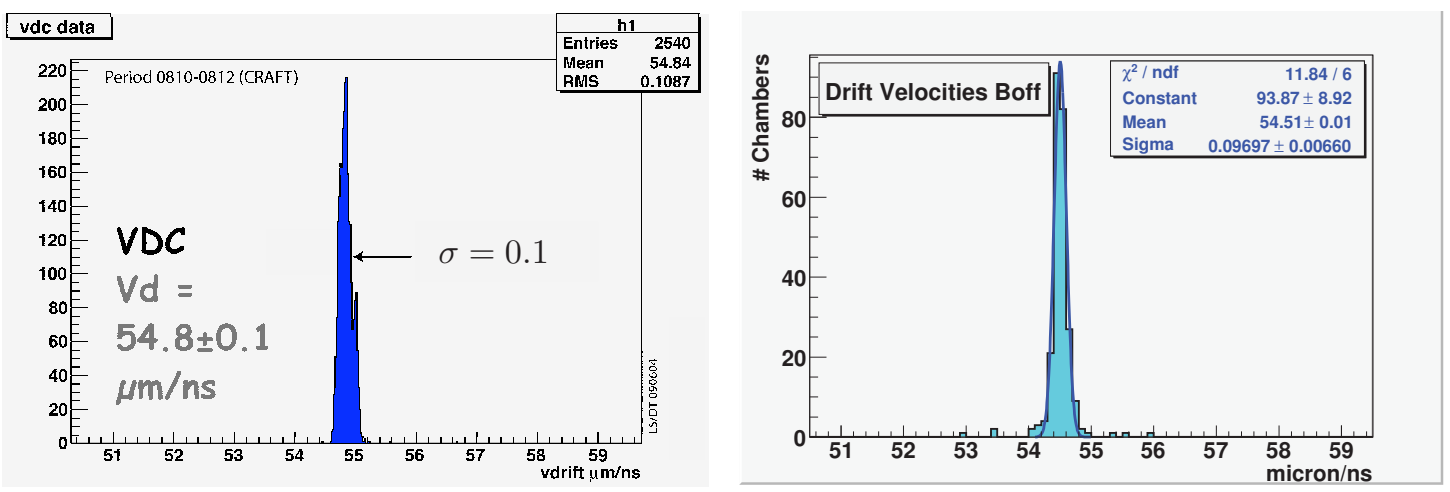

Figure 2: The drift velocity measured with a VDC at CMS (left) in comparison to the drift velocity of the drift tube chambers (right) determined by means of measured cosmic muon tracks with solenoid magnetic field switched off. The plots show average values of the different muon stations and wheels.

(up to $+1900 \mathrm{~V}$ ), cathode (up to $-15000 \mathrm{~V}$ ) and field shaping electrodes. Two electron beams cross a sensitive region between anode and cathode. The sensitive region is characterised by a highly uniform electric field. The electrons are detected by scintillating fibres and two silicon photomultipliers (SiPM's) to provide a trigger. The drift times of the two beams are recorded with respect to the trigger. The average drift time between both parallel beams, separated by $4.8 \mathrm{~cm}$ is being measured as given in Fig. 2, left to $54.8 \mu \mathrm{m} / \mathrm{ns}$ with a width of $0.1 \mu \mathrm{m} / \mathrm{ns}$ by means of a single VDC which is in operation at CMS. The measured drift velocity corresponds to two days of data taking during the cosmic muon run period of CMS in August 2008. This can be compared to the indirectly determined drift velocity of the DT's of $54.5 \mu \mathrm{m} / \mathrm{ns}$ with a width of $0.1 \mu \mathrm{m} / \mathrm{ns}$ obtained from the cosmic muon data taking period 2008. The electric field of the VDC can be varied in a wide range without losing its homogeneity. The VDC measures the drift velocity directly for a given electric field while in the DT's the determined drift velocity is an effective parameter depending beside the gas admixture on the electric field, paths, software selection and fit algorithms. Therefore the drift velocity measured with the VDC can be exploited to verify the gas admixture and the indirectly determined drift velocity of a given muon station.

The complete VDC system consists of two racks. The first rack contains six VDC's (one for each wheel plus a spare one), pressure controllers and sensors, high voltage (HV) for the VDC's, a trigger for each VDC and a crate with dedicated VME modules. The second rack contains VME logics, a readout PC (Linux server), NIM modules, an UPS protection power supply and a gas crate.

Determination of the systematic uncertainties of the drift velocities measured by the six VDC's simultaneously while branched on one single gas circuit are on the way. Furthermore, calibration runs for $\mathrm{HV}$, PM voltage, gas admixtures, impurities $\left(\mathrm{O}_{2}, \mathrm{~N}_{2}\right)$, pressure and temperature dependencies are in the process of being accomplished. In summary, gas admixture anomalies and the drift velocity of the DT's in the muon stations can be verified and validated by means of the VDC monitoring system which measures the drift velocity directly.

\section{References}

[1] CMS collaboration, The CMS experiment at the CERN LHC, JINST 3 (2008) S08004. 